\title{
Une luxation aiguë d'épaule à ne pas réduire aux urgences !
}

\section{All Acute Shoulder Dislocation Should not Be Reduced in the ED!}

\author{
P. Kauffmann $\cdot$ F. Perisse $\cdot$ P. Le Borgne
}

Reçu le 18 septembre 2017; accepté le 11 décembre 2017

(C) SFMU et Lavoisier SAS 2018

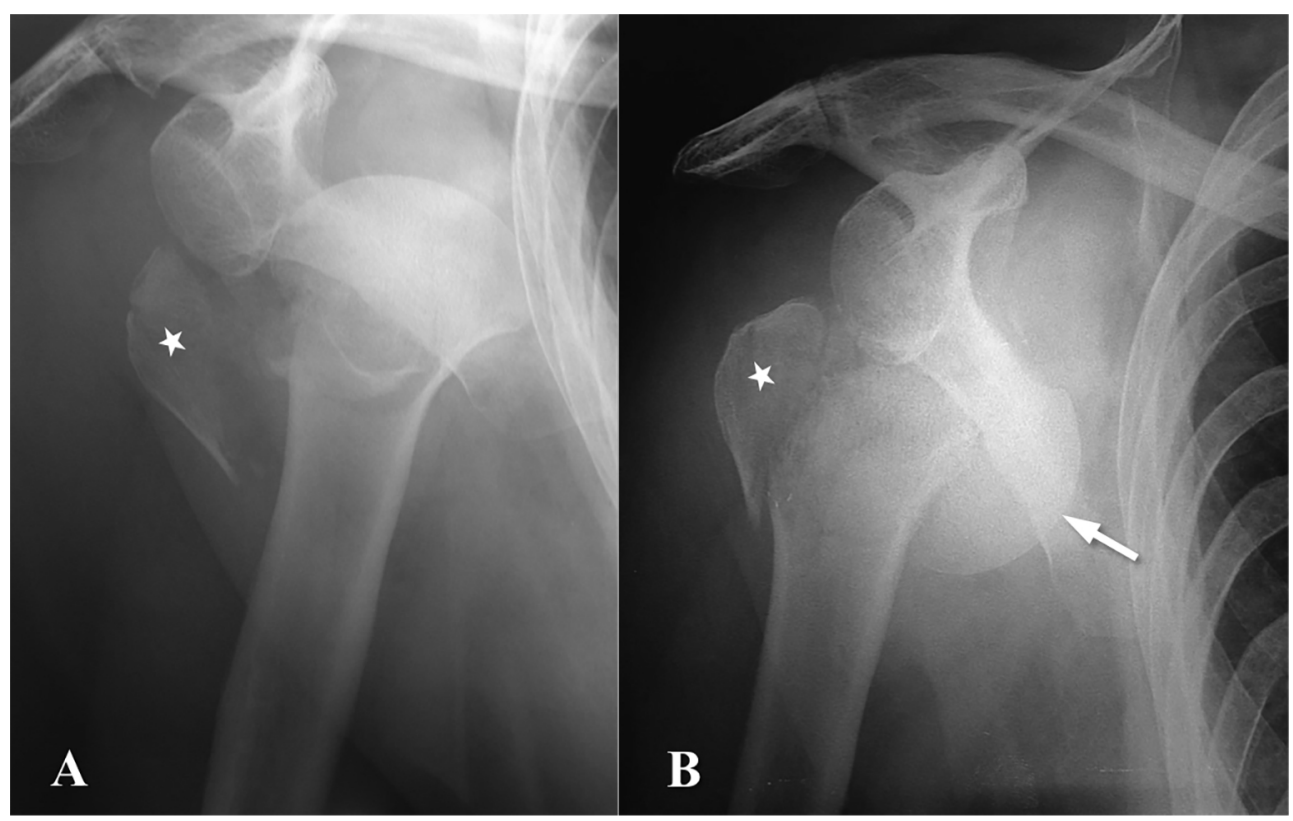

Fig. 1 A. Radiographie initiale de l'épaule droite (face) : luxation glénohumérale antérieure associée à une fracture de la grosse tubérosité (étoile blanche) ; B. Radiographie de l'épaule droite (face) après échec de réduction : vacuité de la cavité glénoïde et apparition d'une fracture déplacée de la tête humérale (flèche blanche)

Un homme de 48 ans s'est présenté aux urgences pour une douleur de l'épaule droite à la suite d'une chute de vélo. À son admission, il présentait l'attitude des traumatisés du membre supérieur et se plaignait d'importantes paresthésies de celui-ci. L'inspection puis l'examen clinique orientaient d'emblée vers une probable luxation antérieure de l'épaule. Il n'existait aucun trouble vasculonerveux associé, mis à part

P. Kauffmann · F. Perisse · P. Le Borgne $(\triangle)$ Service d'accueil des urgences, hôpital de Hautepierre, hôpitaux universitaires de Strasbourg,

1, avenue Molière, F-67098 Strasbourg, France

e-mail : pierrick_med@yahoo.fr

\section{P. Le Borgne}

Faculté de médecine, université de Strasbourg,

4, rue Kirschleger, F-67000 Strasbourg, France ces paresthésies diffuses de l'ensemble du membre qui évoquaient une irritation du plexus brachial. La radiographie de face confirmait la luxation glénohumérale antérieure et retrouvait une fracture associée de la grosse tubérosité de l'humérus (Fig. 1A). Une réduction de la luxation fut alors entreprise aux urgences dans le cadre d'une sédation procédurale (kétamine + hypnovel). La radiographie de contrôle montrait toutefois l'échec de la réduction et surtout le détachement de la tête humérale de sa diaphyse avec un déplacement médial dans l'aisselle (Fig. 1B). La luxation glénohumérale antérieure est une pathologie fréquente aux urgences. Même si l'examen clinique permet habituellement d'affirmer la luxation, il est licite de réaliser une radiographie dans le bilan initial, afin de rechercher des lésions associées, notamment une fracture de la grosse tubérosité de l'humérus. Cette fracture est fréquente (jusqu'à $35 \%$ des 
luxations antérieures), avec une incidence qui augmente avec l'âge des patients (au-delà de 40 ans) [1]. Elle est générée par l'action conjointe de deux éléments : la traction, au moment de la luxation, des tendons de la coiffe des rotateurs qui s'y insèrent et l'impaction du rebord glénoïdien inférieur sur l'humérus. Une fracture volumineuse de la grosse tubérosité rend l'épiphyse humérale proximale plus fragile. En sa présence, les manœuvres de réduction peuvent donc être responsables de la survenue d'une fracture iatrogène, soit par le déplacement d'une fracture non visible sur la radiographie initiale, soit par le « décapsulage » de la tête humérale contre le rebord glénoïdien au moment de la manœuvre. Il apparaît alors une fracture-luxation de l'humérus proximal présentant plusieurs fragments (dont la tête humérale) qui peuvent évoluer vers la pseudarthrose, le cal vicieux et/ou l'ostéonécrose en raison de l'importance de leur dévascularisation (lésions des attaches capsuloligamentaires). Les patients de plus de 40 ans présentant un premier épisode de luxation antérieure, associé à une fracture de la grosse tubérosité, présenteraient un risque significatif (environ $5 \%$ ) de fracture iatrogène lors de la tentative de réduction, malgré une sédation adéquate [2]. S'il existe un doute de fracture associée du col huméral sur la radiographie initiale (contre-indiquant de facto la réduction aux urgences), un scanner osseux pourra alors permettre d'affiner le diagnostic lésionnel. En dehors de ce cas de figure, il est possible de réaliser les manœuvres de réduction, en présence du chirurgien et sous parfaite analgésie et relâchement musculaire (idéalement au bloc opératoire). À la suite de cette complication du geste de réduction, notre patient a bénéficié d'une intervention chirurgicale en urgence, qui a permis la réduction à foyer ouvert de la luxation et l'ostéosynthèse des différents éléments osseux fracturés.

Liens d'intérêts Les auteurs déclarent ne pas avoir de liens d'intérêts.

\section{Références}

1. Bencardino JT, Gyftopoulos S, Palmer WE (2013). Imaging in anterior glenohumeral instability. Radiology. 269:323-37

2. Atoun E, Narvani A, Even T et al (2013). Management of firsttime dislocations of the shoulder in patients older than 40 years: the prevalence of iatrogenic fracture. J Orthop Trauma. 27:190-3 